\title{
Labyrinthe
}

$20 \mid 2005(1)$

La Cognition

\section{Evelyn Fox Keller, Expliquer la vie : modèles, métaphores et machines en biologie du développement}

Gallimard, 2004, 416 p., [Titre original : Making Sense of Life, Explaining Biological Development with Models, Metaphors and Machines,

Cambridge, MA: Harvard University Press, 2002. Traduit de l'anglais par Stéphane Schmitt]

\section{Charles Ruelle}

\section{(2) OpenEdition}

\section{Journals}

Édition électronique

URL : http://journals.openedition.org/labyrinthe/771

DOI : 10.4000/labyrinthe.771

ISSN : 1950-6031

Éditeur

Hermann

Édition imprimée

Date de publication : 20 avril 2005

Pagination : 167-171

Référence électronique

Charles Ruelle, «Evelyn Fox Keller, Expliquer la vie : modèles, métaphores et machines en biologie du développement », Labyrinthe [En ligne], 20 | 2005 (1), mis en ligne le 09 juillet 2008, consulté le 02 mai 2019. URL : http://journals.openedition.org/labyrinthe/771 ; DOI : 10.4000/labyrinthe.771 


\title{
Expliquer la vie: modèles, métaphores et machines en biologie du développement d'Evelyn Fox Keller*
}

\author{
Charles RuELle \\ charles.ruelle@freesbee.fr
}

Evelyn Fox Keller (née en 1936) est sans doute aujourd'hui l'une des figures les plus atypiques de l'histoire des sciences. Formée à la physique théorique, reconvertie à la biologie moléculaire, elle s'engage dès les années 1970 dans le mouvement féministe américain. La publication, en 1974, d'un premier article sur la place des femmes dans la société scientifique ${ }^{1}$ l'entraîne sur la voie des Gender Studies qui cerneront dès lors le cadre théorique d'une partie de ses études sur la science. Toutefois, dans sa quête des dimensions subjectives de l'activité scientifique, E. F. Keller semble privilégier depuis quelques années des pistes parallèles. Il en est ainsi dans son livre Refiguring Life: Metaphors of Twentieth Century Biology (1995) où elle tente d'analyser la portée effective des métaphores scientifiques - avec ce qu'elles recèlent de culturel et de métaphysique - pour illustrer le rôle du langage dans la science. Ironie de la démarche, c'est par l'exposition du concept d' «énoncé performatif» de J. L. Austin² que E. F. Keller aborde son ouvrage. Représentant éminent de la philosophie analytique d'Oxford, Austin se situe en effet à l'opposé des positions vaguement qualifiées de postmodernes d'E. F. Keller, dont il aurait sans aucun doute rejeté avec force les principes ${ }^{3}$. Pour affirmer son ambition de

* Gallimard, 2004, 416 p., [Titre original : Making Sense of Life, Explaining Biological Development with Models, Metaphors and Machines, Cambridge, MA: Harvard University Press, 2002. Traduit de l'anglais par Stéphane Schmitt].

1. «Women in Science. An Analysis of a Social Problem », Harvard Magazine, octobre, 1974, p. 14-19.

2. John L. Austin, Quand dire c'est faire, Seuil, 1991 [1962].

3. La philosophie analytique se donne pour objet la clarification logique de la pensée. Bien qu'elle ait en partie renoncé à la «conception scientifique du monde» des positivistes logiques, elle garde un rapport privilégié à la science. E. F. Keller, qui entend dépasser le logicisme de la pensée, tient 
décloisonner les savoirs, les cultures et de réconcilier la science avec ses détracteurs, E. F. Keller ne pouvait pas mieux s'y prendre.

Dans son dernier ouvrage, Expliquer la vie: modèles, métaphores et machines en biologie du développement, E. F. Keller prend une nouvelle fois le parti de l'analyse linguistique et poursuit l'exploration des mécanismes de «l'explication scientifique» au $\mathrm{XX}^{\mathrm{e}}$ siècle. Son ambition est alors clairement de renouveler le champ d'analyse d'un concept largement laissé jusqu'ici à l'appréciation de philosophes issus de l'empirisme logique pour qui «la validité (et/ou la puissance) de l'explication va de soi en science» (p. 14). Les questions posées sont les suivantes: "Comment peut-on réellement savoir ? Qu'est-ce qui, de fait, a valeur d'explication? Et de théorie?» (p. 8). Sur ce point, la position de l'auteur est claire : «Les réponses à de telles questions sont non pas nécessaires, mais contingentes, non pas universelles, mais affaire de culture disciplinaire locale et historiquement spécifique» (p. 8 ). Toute explication semble ainsi dépendre des outils techniques et conceptuels dont le scientifique dispose lors de son étude, ainsi que des besoins individuels ou collectifs qui orientent sa recherche. Tous ces facteurs génèrent des procédures diversifiées qui empêchent de concevoir la science comme un domaine unifié. Cette thèse s'avère, en fin de compte, servir de fondement à la défense d'un pluralisme théorique qui reconnaît la nécessité de faire intervenir une pluralité de causes, de modèles et d'hypothèses pour rendre compte de la complexité des phénomènes ou combler les limites de notre entendement à les embrasser dans leur totalité. Résolument empirique, l'étude s'appuie sur l'histoire de l'embryologie au $\mathrm{XX}^{\mathrm{e}}$ siècle. E. F. Keller tente d'exhiber la diversité des «types de discours» utilisés successivement pour expliquer le développement. Tous correspondent à des stratégies explicatives différentes pour lesquelles les modèles, les métaphores ou les machines tiennent respectivement une place prépondérante.

La première partie du livre examine ainsi un certain nombre de tentatives de la première moitié $\mathrm{du} \mathrm{XX}^{\mathrm{e}}$ siècle pour expliquer la forme biologique en recourant à des modèles physico-chimiques ou mathé-

une position plus proche des philosophes postmodernes qui prônent « la fin des grands récits » et l'abandon d'une philosophie reposant sur une rationalité unitaire, voire purement logiciste. Il est vrai, toutefois, que Keller et Austin tiennent des positions respectives relativement modérées par rapport au postmodernisme et au positivisme. Cela autorise certains rapprochements. 
matiques «sans l'aide des gènes», tels ceux de S. Leduc, W. d'Arcy Thompson, N. Rashevsky ou A. Turing. Le chapitre consacré à Leduc s'avère particulièrement passionnant et représentatif de l'explication par le biais de modèles. Leduc tentait «d'expliquer l'émergence de la forme biologique en synthétisant des organismes artificiels à partir de substances chimiques inorganiques» (p. 21). À ces fins, il reproduisait artificiellement des phénomènes de diffusion et de croissance osmotique en faisant réagir ensemble des substances inorganiques. La similitude des organismes avec les formes ainsi synthétisées devait servir de principe explicatif de l'ontogenèse. Dans le cadre d'une biologie du développement peu encline à accepter le rôle de l'ADN, un tel modèle pouvait suffire à expliquer le développement. L'explication scientifique n'avait pas à prendre en compte une cause interne à l'organisme pour en expliquer le devenir. Toutefois, la prise de conscience de l'implication du gène dans les phénomènes ontogéniques a, dès les années 1930 , réorienté les scientifiques vers l'analyse des processus biologiques effectivement à l'œuvre dans les organismes. À ce compte, la portée explicative ne relevait plus simplement de la similitude d'un modèle à son substrat biologique, mais de la capacité métaphorique d'un concept à exprimer l'action causale du gène dans le développement. C'est l'ensemble de ces mutations qu'E. F. Keller analyse dans sa seconde partie «Les métaphores: gènes et récits du développement».

Dans son ouvrage précédent, Le Siècle du gène, E. F. Keller avait déjà tenté de montrer que l'évolution de la signification du mot "gène » - depuis la définition de H. J. Muller comme «base de la vie ${ }^{4} »$ jusqu'à la remise en cause des dogmes de la biologie moléculaire - était la preuve de son imprécision conceptuelle. La multiplicité de sens du mot «gène» devait alors mettre en doute son caractère opératoire. Ici, en revanche, E. F. Keller obtient des résultats inverses et «plaide en faveur du caractère productif de l'imprécision linguistique» (p. 345, note 7). «L'absence d'un sens clair et univoque du concept de gène lui-même, c'est-à-dire de l'unité explicative fondamentale, peut constituer - nous dit-elle - une ressource positive permettant de réunir différents systèmes expérimentaux et différents programmes de recherche en un projet scientifique cohérent» (p. 139). Il reste que depuis une dizaine

4. Hermann J. Muller, « The Gene as the Basis of Life », Proceedings of the International Congress of Plant Science, 1, p. 897-921. 
d'années, l'introduction de nouvelles techniques dans l'analyse des données de la biologie moléculaire tend à transformer notre rapport à l'organisme, dont la simple faculté à s'adapter au sens d'une métaphore ne suffit plus à expliquer le développement. La découverte de nouvelles techniques d'imagerie moléculaire depuis les années 1970 semble transformer nos critères épistémologiques d'explication.

D'une part, l'introduction de techniques de visualisation des processus moléculaires intracellulaires (marqueurs $\mathrm{GFP}^{5}$, microscopie vidéo $\mathrm{CLSM}^{6}$ ) a permis la renaissance d'une épistémologie visuelle héritée des $\mathrm{XVIII}^{\mathrm{e}}$ et XIX ${ }^{\mathrm{e}}$ siècles comme premier fondement de l'explication. Secondement, l'apparition de machines ultra puissantes a renforcé, au sein des normes explicatives, la nécessité de faire appel à des modèles mathématiques et informatiques pour analyser l'énorme quantité de données rendues accessibles par ces machines. Dans ce cas, ce qui est compréhensible, c'est ce qui est modélisé par le biais d'instruments d'analyse. Ou autrement dit: expliquer, c'est se fier à l'analyse des machines. Enfin, d'autres machines propres selon leur concepteur à reproduire la vie ont introduit une dimension «pragmatique» de l'explication (p. 223) qui met celle-ci sur la voie de la fabrication, de la «simulation» comprise comme une imitation. Qu'on se souvienne seulement de cette phrase éloquente de Steven Levy: «C'est en fabriquant la vie que nous saurons enfin ce qu'est la vie ${ }^{7} »$. Ce n'est assurément pas la position d'E. F. Keller qui remarque justement qu'on ne peut pas prétendre découvrir ce qu'est la vie en la rapprochant d'une classe d'objets, sans présupposer qu'elle est de la même nature que cet objet. Si cela était possible, il faudrait aussi supposer que l'on puisse faire de la vie une catégorie naturelle et qu'elle est moins le produit d'une histoire que d'une évolution. E. F. Keller se refuse à le penser et constate avec Michel Foucault que «la vie est une catégorie humaine plus que naturelle. Tout comme l'explication ${ }^{8} »$ (p. 320).

5. GFP pour Green Fluorescent Protein.

6. CLSM pour Confocal Laser Scanning Microscopy.

7. Voir Artificial Life. The Quest for a New Creation, New York, Pantheon Books, p. 10. (Cité par E.

F. Keller, op. cit., p. 316).

8. Foucault défend en effet la thèse que la vie - en tant que concept et objet d'étude - est une «invention » culturelle qui date du XIX ${ }^{\mathrm{e}}$ siècle. Ainsi écrit-il: «On veut faire des histoires de la biologie au $\mathrm{XVIII}^{\mathrm{e}}$ siècle ; mais on ne se rend pas compte que la biologie n'existait pas [...]. Et si la biologie était inconnue, il y avait à cela une raison bien simple: c'est que la vie elle-même n'existait pas. », dans Les Mots et les choses, Paris, Gallimard, 1966, p. 139. 
Malgré la sagesse de cette observation, on ne peut se satisfaire sans réserve de la thèse générale de cet ouvrage, qui met en place un relativisme déguisé et contradictoire que l'auteur ne semble pas vouloir assumer. La dépendance de l'explication à l'égard de besoins tant collectifs qu'individuels semble en effet bien difficile à concilier avec l'objectivité - même imparfaite - que l'on attend de toute explication scientifique. $\mathrm{Si}$, en outre, le pouvoir explicatif d'un discours ne correspond à rien d'autre qu'à la satisfaction personnelle qu'on en tire ${ }^{9}$, alors on réduit l'appréciation de la vérité, la compréhension, à un jugement esthétique. Le vrai, comme le beau, est réduit à la simple expression du chercheur en situation ${ }^{10}$. À ce titre, on ne voit pas comment E. F. Keller peut éviter de faire de la science une activité potentiellement ultra individualisée - en contradiction avec l'existence de cultures épistémologiques, même locales - et échapper à un relativisme extrême. Est-ce là l'unique destinée du pluralisme théorique et de l'interdisciplinarité? Assurément pas. Du moins devons-nous faire en sorte qu'il en soit autrement ${ }^{11}$.

9. «Ce qui conduit celui ou celle qui étudie les processus à s'exclamer "Eurêka!" dépend de ses attentes, de ses besoins et de ses désirs. », dans E. F. Keller, Expliquer la vie..., p. 326.

10. Ludwig Wittgenstein, «Leçons sur l'esthétique », dans Leçons et Conversations, Paris, Gallimard, 1971, p. 15-78.

11. On ne peut que renvoyer ici à l'ouvrage de Sandra Mitchell, Biological Complexity and Integrative Pluralism, Cambridge Studies in Philosophy and Biology, Cambridge (UK): Cambridge University Press, 2003. 